\title{
With a little help from my peers: Depicting social norms in a recommender interface to promote energy conservation
}

\author{
Alain Starke ${ }^{\dagger}$ \\ Department of Information \\ Science \& Media Studies, \\ University of Bergen, Norway \\ Alain.Starke@uib.no
}

\author{
Martijn C. Willemsen \\ Jheronimus Academy of Data \\ Science, Eindhoven University of \\ Technology, the Netherlands \\ M.C.Willemsen@tue.nl
}

\author{
Chris Snijders \\ Human-Technology Interaction \\ Group, Eindhoven University of \\ Technology, the Netherlands \\ C.C.P.Snijders@tue.nl
}

\begin{abstract}
How can recommender interfaces help users to adopt new behaviors? In the behavioral change literature, nudges and norms are studied to understand how to convince people to take action (e.g. towel re-use is boosted when stating that ' $75 \%$ of hotel guests' do so), but what is advised is typically not personalized. Most recommender systems know what to recommend in a personalized way, but not much research has considered how to present such advice to help users to change their current habits. We examine the value of presenting normative messages (e.g. ' $75 \%$ of users do X') based on actual user data in a personalized energy recommender interface called 'Saving Aid'. In a study among 207 smart thermostat owners, we compared three different normative explanations ('Global', 'Similar', and 'Expert' norm rates) to a non-social baseline ('kWh savings'). Although none of the norms increased the total number of chosen measures directly, we show evidence that the effect of norms seems to be mediated by the perceived feasibility of the measures. Moreover, how norms were presented (i.e. specific source, adoption rate) affected what measures were chosen within our Saving Aid interface.
\end{abstract}

\section{CCS CONCEPTS}

- Information Systems $\rightarrow$ Decision Support Systems

- Human-centered Computing $\rightarrow$ User Studies

\section{KEYWORDS}

\section{Recommender Systems, Behavioral Change, Energy Conservation, User Experience, Rasch Model}

\section{Introduction}

Changes in a choice architecture that lead to predictable behavior are 'nudges' [54]. Prominent examples are default choices [28], and the use of normative messages ('most users do $\mathrm{X}^{\prime}$ ) [22]. The use of nudges and persuasive messages is rather uncommon in personalization domains as recommender systems.

Permission to make digital or hard copies of part or all of this work for personal or classroom use is granted without fee provided that copies are not made or distributed for profit or commercial advantage and that copies bear this notice and the full citation on the first page. Copyrights for third-party components of this work must be honored. For all other uses, contact the owner/author(s). IUI'20, March, 2020, Cagliari, Italy

(c) 2020 Copyright held by the owner/author(s). XXXXXXXXXXXXXXXX. https://doi.org/XX.XXXX/XXXXXXXX
While recommenders typically provide decision support by optimizing what to recommend [27, 40], nudges focus on how such content should be presented. This way, nudges can shift a user preferences, which is illustrated by studies on explanations in recommender interfaces $[11,55]$. For example, if a recommender explains that a user's peers have chosen specific items, this might steer user preferences towards these items, even if they have a worse fit according to the recommender system [11].

Nudges could be particularly important in domains where selfactualization and behavioral goals play a role [17], as users often consider what their peers are doing [13, 39]. In recent years, energy and health recommender systems have emerged [35, 44, 51], in which users seek to change their lifestyle, attaining new goals that typically require them to step away from their current preferences and choose new items [44,51]. Research shows this is hard, since the personalized recommendations offered by a system vary substantially in terms of the required effort and benefits [ 4 , $10,20,31,57]$. Users often choose the easier recommendations [51], instead of the ones that reap the most benefits (e.g. have the largest environmental impact). Moreover, personalization algorithms that promote popular items (e.g. collaborative filtering), are possibly inadequate to support behavioral change, for they reinforce a user's current habits [17, 45, 50].

The addition of nudges may be effective to persuade users to take the most beneficial recommendations. Nudges can explain why an item is recommended [55], highlight specific attributes of recommendations (e.g. kWh) [25, 37], and show the behavior of other relevant users $[9,11,34]$. However, it is unclear to what extent such norms are still effective when presented in a list that is already tailored towards a user's preferences. Previous studies have suggested that the impact of one-size-fits-all persuasion might be lost on tailored interventions [33, 51].

Furthermore, most nudges are not yet personalized [21]. This could cause problems, as some persuasive messages only appeal to specific users [33, 47], and can lead to unintended behavior among other users [12]. For instance, a food recommender system that explains ' $80 \%$ of users also bought this pack of cookies', could steer users away from their healthy eating goals and stifle behavioral change. Conversely, if norms accentuate that only few users perform a certain behavior, they can deter others from showing that same behavior $[12,54]$. For example, solar PV has only been installed on $9 \%$ of Dutch houses [29], which is not very convincing when presented as a normative message. 
The current paper blends the domains of nudging and recommender systems to help users attain their energy-saving goals. We focus on social explanations to signal a majority norm, affecting user preferences through social proof $[13,18]$. Since such majority norms are hard to achieve for behaviors that are only performed by a small part of the general population (e.g. solar PV), we explain energy-saving measures in terms of specific population groups with higher adoption rates (e.g. expert users). [7, 31]. For example, the adoption rate of Solar PV among more experienced users is much higher than the average rate of $9 \%$ [29, 52 ], and possibly exceeds $50 \%$ among users with a strong energysaving attitude [51].

This is the point of departure in this paper. By considering specific peer groups in a normative intervention, we design an energy recommender interface named 'Saving Aid'. In a web study, we assess the energy-saving attitude of each user through the psychometric Rasch model, and present attitude-tailored advice subsequently. In addition, we use the Rasch model to craft different social norms of peer groups with specific attitudes ('Similar' and 'Experienced' norms), which might have higher adoption rates than the general population ('Global' norms'), and compare their effectiveness to a non-social baseline condition. We posit the following research question:

[RQ]: To what extent can descriptive norms boost the adoption of a heterogeneous set of tailored energy-saving measures?

\section{Literature review and hypotheses}

This review discusses the mechanisms of descriptive norms in psychological literature, contextualizes them in the HCI domain, and formulates hypotheses for the proposed web study. It explains how the psychometric Rasch model is used to craft effective social norms, which are used in our 'Saving Aid' recommender interface.

\subsection{Descriptive norms}

To date, energy recommender interfaces $[35,51,56]$ have neglected that conservation decisions are not made in a social vacuum [1, 43]. The field of HCI can learn from studies in environmental psychology, which provide theoretical and empirical evidence that explaining behaviors in terms of relevant peer groups [24, 49] and descriptive norms [22, 24, 43, 49] can affect one's energy-saving behavior and decision-making.

A notable finding is that highlighting descriptive norms can outperform environmental explanations of sustainable behavior [22]. Showing that a rather large proportion of peers performs a certain behavior [13, 24, 38], can trigger a descriptive norm that promotes socially-desirable behavior [11, 60]. Two mechanisms underlie this effect: compliance (i.e. the propensity to act consistent with presented norms) and conformity (i.e. adapting one's behavior to match an apparent majority) [14].

Goldstein et al. [22] show that hotel guests are more inclined to re-use their towels when asked to do so using descriptive norms ('join your fellow guests in helping to save the environment'), compared to a general environmental message ('help to save the environment'). Such normative messages highlight a community aspect (' $75 \%$ of guests participated'), and are more convincing if they include context-rich or 'local' aspects [13, 22]. For instance, referring to ' $75 \%$ of hotel guests', rather than ' $75 \%$ of citizens' is more effective, for it highlights an uncommon characteristic with the decision-maker $[19,22,26]$.

Rather than boosting a specific behavior, descriptive norms are also used to promote a wider range of sustainable behaviors [41]. For example, customers of web shops purchase more healthy and energy-saving products, if they are explained using social norms instead of their environmental impact $[3,15]$. We hypothesize that this also applies to our personalized recommender interface:

[H1]: Explaining energy-saving measures using descriptive norms rather than through appeals on environmental impact increases the probability of those measures being selected.

\subsection{Rasch model}

There is arguably a large range of norm percentages (probably anything below 50\%) that might not trigger conformity [11, 13, 14]. Although it is hard to promote 'unpopular' measures as 'Install Solar PV' [51], such measures typically yield relatively high kWh savings [53]. Therefore, it might pay off to somehow promote them by making them stand out in the larger set of personalized user recommendations.

The dimensionality of energy-saving behavior illustrates the large variety in adoption rates between measures [10, 31]. Research in environmental psychology shows that energy-saving measures can be mapped on a one-dimensional scale using the psychometric Rasch model, based on how often these measures are performed [57]. In the context of attitude theory 'Campbell's Paradigm' [31], this adoption rate is operationalized as behavioral costs, which represents the execution difficulty of a measure and comprises different types of costs, such as money, time, and cognition [58]. This approach postulates that measures with smaller adoption rates face higher behavioral costs. For example, a study that fitted a Rasch scale of 79 energy-saving measures, shows that $92 \%$ of respondents lower the thermostat when leaving the house for a longer period [51], which has a relatively low behavioral cost level, while only $7 \%$ of respondents uses an energy-efficient heat pump, which has high behavioral costs.

The characteristics of the Rasch model can be used to craft convincing social norms. An HCI study on energy recommender systems by Starke et al. [51] shows how to form a latent factor model by asking a group of persons to indicate whether they perform a set of energy-saving measures, or not [31, 57]. Besides ordering measures on their adoption rates this way, users are also ordered with respect to how many measures they perform, which is operationalized as a person's energy-saving attitude [31]. Users with stronger attitudes are assumed to perform more measures.

The adoption rates of measures are what we label 'Global' norms. These are statements about the general population that can be presented alongside energy-saving recommendations, analogous to the norms used by Goldstein et al. [22]. For example, " $55 \%$ of other users has installed weather strips on doors" [53]. As discussed in the introduction, we expect normative messages as " $75 \%$ of participants use $X$ " to signal that the majority of a population has already adopted a certain energy-saving measure, 
and are therefore expected to be more persuasive than minority norms, such as " $30 \%$ of users do X." We hypothesize the following:

[H2]: Energy-saving measures are more likely to be selected from a recommendation list when presented with higher rather than low norm adoption rates.

\subsection{Crafting 'local' personalized social norms}

The Rasch model is also used to infer a person-dependent probability that a measure is already performed. Eq. 1 shows that the probability $p$ that a measure $i$ is performed not only depends on a measure's behavioral costs $\delta$, but also on the attitudinal strength $\theta$ of individual $n$, where $\delta$ and $\theta$ are expressed in logistic scale units (logits) [31, 57]:

Prob $\{n$ performs measure $i\}=\ln \left(\frac{p_{n i}}{1-p_{n i}}\right)=\theta_{n}-\delta_{i}$

For any energy-saving measure, Rasch predicts the same adoption probability for all users with a specific attitudinal strength [32], as well as increasing probabilities for users with stronger attitudes. Among the larger population, we consider this probability to be the adoption rate. This allows us to craft personalized social norms that compare against peer users with similar or higher attitudinal strengths, which might be more effective than a non-personalized global norm for some measures.

In addition to the norm adoption rate, literature on advicetaking shows that the specific peer group also influences choice and acceptability [7], suggesting two important characteristics. First, similarity in relevant attitudes can increase the extent to which advice is considered or liked [7, 48]. The Rasch scale allows the design of 'Similar' norms alongside recommendations, which can show higher adoption rates than global norms, especially for users with stronger attitudes. For example, users with a strong attitude might be presented the 'Global' norm " $20 \%$ of users have installed radiator reflectors" [51], while the 'Similar' norm would be " $60 \%$ of users like you have installed radiator reflectors".

A second characteristic is a peer's perceived expertise [7, 30]. Expert advice is less likely to be ignored than suggestions from novices $[6,7,30]$. In this study, we assume peers to possess such higher expertise if they perform more measures ('Experienced' norms), thus having stronger attitudes and higher adoption rates. For example, where 'Similar' norms would report " $55 \%$ of users like you do X", 'Experienced' norms at an attitude $\theta$ that is +1 logit stronger than the user report an adoption rate of $78 \%$.

Combining different advice sources and adoption rates, we craft three different normative messages:

- Global norms: "X\% of users perform this measure."

- Similar norms: "Y\% of users who perform similar measures as you, perform this measure."

- Experienced norms: " $Z \%$ of users who perform more measures than you, perform this measure."

\subsection{Global vs. local norms}

To discuss the effectiveness of each norm, we consider a recommendation scenario. Suppose there are two users, and that User 1 has a weaker attitude $\left(\theta_{1}=-1\right)$ than User $2\left(\theta_{2}=+1\right)$. If both users are presented a measure with behavioral costs $\delta$ equal to their attitude $\theta$, then User 1 is shown a measure with lower behavioral costs than User 2 .

Table 1 outlines the presented peer adoption rates of this scenario. The adoption rates for 'Global' norms depend on the user's attitude. User 1 has a relatively weak attitude, and therefore her attitude-tailored measure has a high 'Global' adoption rate (i.e. 70\%). In contrast, User 2 has a stronger attitude and, therefore, her attitude-tailored measure has a lower 'Global' adoption rate of $30 \%$, which is not very convincing. The adoption rates of the personalized 'Similar' or 'Experienced' norms do not depend on the global adoption rate and are identical for both users (resp. 50\% and $75 \%$ ) and thus potentially more convincing for User 2.

Table 1. Recommendation scenario for two users with different energy-saving attitudes. Cell entries show which percentage would be shown to users in each scenario.

\begin{tabular}{|c|c|c|c|}
\hline & \multicolumn{3}{|c|}{$\begin{array}{c}\text { Presented \% of peer adoption rate for } \\
\text { attitude-tailored measures }\end{array}$} \\
\hline & Global & $\begin{array}{l}\text { Similar } \\
(=\text { User } \theta)\end{array}$ & $\begin{array}{l}\text { Experienced } \\
(=\text { User } \theta+1)\end{array}$ \\
\hline User 1: $\theta_{1}=-1$ & $72 \%$ & $50 \%$ & $75 \%$ \\
\hline User 2: $\theta_{2}=+1$ & $30 \%$ & $50 \%$ & $75 \%$ \\
\hline
\end{tabular}

We use Table 1 to formulate hypotheses. Based on the adoption rates, we would expect users with stronger attitudes (User 2) to choose more measures when facing 'Similar' norms, while users with weak attitudes (User 1) would do so for 'Global' norms. However, the higher degree of similarity for 'Similar' compared to 'Global' norms could mitigate the adoption rate's appeal [22, 43]. Nonetheless, another study [59] argues that inexperienced users (e.g. with a weak attitude) are more likely to rely on majority advice than similarity (e.g. a 'Global norm \%'), while experienced individuals (e.g. a strong attitude) rely on similar peers. Based on these arguments, we formulate the following hypotheses:

[H3a]: Users with a strong energy-saving attitude are more likely to select measures from a recommendation list, if they are explained using similar norms rather than global norms.

[H3b]: Users with a weak energy-saving attitude are more likely to select measures from a recommendation list, if they are explained using global norms rather than similar norms.

Table 1 also suggests an additional benefit of higher adoption rates for 'Experienced' norms compared to 'Similar'. However, their persuasiveness may be mitigated because of their reduced similarity ("others who perform more measures than you"). Nonetheless, we expect that the higher adoption rates for Experienced norms (75\%) across an entire recommendation list will be more persuasive, particularly for the adoption of complicated or effortful items or measures [59]. We posit the following hypothesis:

[H4]: Users are more likely to select energy-saving measures when facing 'experienced' norms rather than 'similar' norms. 


\subsection{Perceptions of descriptive norms}

Besides evaluating behavior, it is also useful to understand how users perceive such a descriptive norm. Studies in environmental psychology teach us that how an individual evaluates environmental aspects can determine behavioral outcomes [2, 16]. For instance, social proof of others performing a particular behavior might lower the thresholds towards performing it [18].

Previous recommender studies similarly have highlighted the importance of perceptions in explaining the user experience [36], as these allow us to understand why a change in a particular system aspect increases the user experience. For example, Starke et al. [51] showed that tailored recommendation lists with low levels of behavioral costs are more likely to be perceived as feasible and, in turn, show stronger perceived support, higher levels of user satisfaction and more energy-efficient choices [51]. Similarly, we expect descriptive norms to lower behavioral thresholds to choose and, eventually, adopt energy-saving measures, which is assessed through perceived feasibility, perceived support and subsequent user satisfaction. We formulate the following hypotheses:

[H5]: Users perceive energy-saving measures that are explained using social norms as more feasible and more supportive, compared to those that emphasize the environmental impact.

[H6]: Perceived feasibility positively affects perceived support.

[H7]: Perceived feasibility and perceived support positively affect choice satisfaction.

[H8]: Higher levels of perceived support lead to more energyefficient choices.

\section{Method}

We have investigated to what extent descriptive norms boost the adoption of a heterogeneous set of tailored energy-saving measures. To do so, we first explain how we validated our onedimensional construct by collecting data in a pre-study. Thereafter, we elaborate on our web study and the design of our energy recommender interface called 'Saving Aid'.

\subsection{Pre-study to set up a Rasch scale for personalized norms}

To generate recommendations based on the Rasch model, we designed a survey that was part of different study [Anonymized reference]. Participants were invited to fill out a short survey on their current energy-saving behavior, and indicated for 13 to 25 randomly sampled energy-saving measures (out of a database of 134) whether they performed them or not ('yes' or no').

We used dichotomous responses from 555 participants $(50.6 \%$ male) with a mean age of 43.4 years $(S D=19.7)$ to fit a onedimensional measurement scale of 134 energy-saving measures. The scale's item parameters were determined reliably $(\alpha=.95, M$ $=.05, S D=1.57$ ), as all measures fitted the construct by meeting the prescribed 'infit' criteria [8]. Due to an item separation of 4.51, we could reliably discern 4 to 5 strata of behavioral costs. In terms of adoption rates, the scale ranged from $94 \%$ to $1 \%$.
In addition, we also inquired how effortful participants perceived measures to be. For each measure separately, we presented a 4-point Likert scale that ranged from 'not effortful' to 'very effortful'. The mean response per measure was added to our analysis as a control variable, labeled as 'perceived effort'.

\section{2 'Saving Aid' recommender study}

Following our pre-study, we set up a web study in collaboration with a Dutch energy supplier. We invited owners of a smart thermostat use our 'Saving Aid' recommender system to find and select appropriate energy-saving measures to take in the household, and to complete our user experience questionnaire.

\subsubsection{Participants}

In total, 217 participants finished the online study, from which we excluded ten for either completing the study in under 3 minutes, indicating to not trust the website, or showing no variation in the evaluation questionnaire.

Eventually, we considered a sample of 207 participants $(M=$ 53.5 years, $S D=14.0)$ that comprised predominantly males $(87 \%)$. Among our participants, only $26.6 \%$ owned the home they lived in, while the majority lived in a town house (58.5\%).

\subsubsection{Procedure}

To estimate each user's attitude, we randomly sampled 13 energy-saving measures from across the behavioral costs scale. These were presented sequentially to users, who indicated whether they performed them or not ('yes' or 'no'). Subsequently, we inquired on the user's housing situation to filter irrelevant measures from the recommendation list.

Afterwards, we presented each user a list of nine energy-saving recommendations, whose behavioral costs were tailored towards the user's estimated energy-saving attitude $(\theta \approx \delta)$. In addition, the measures were ordered on their estimated $\mathrm{kWh}$ savings.

Figure 1 depicts the top two measures of a list, presenting a measure's name, a short description, and a score or percentage accompanied by an explanation. Recommendations were sampled between the adoption probabilities of $18 \%$ to $75 \%$, which ranged from -1.5 to +1 logit in terms of the attitude-behavioral costs difference.

To test our hypotheses, we asked users to select any number of recommended measures that they wished to perform. Users could hover for 'more info' to see other commonly used attributes of an energy-saving measure, such as its frequency and kWh savings. Figure 1 portrays this on the left-hand side of the top measure.

For our hypotheses on the perception of recommendations [H5-H8], we inquired on the user's experience with the system. We presented statements using 7-point Likert scales, as validated in previous studies $[35,51]$, assessing the perceived feasibility of the recommended measures, the perceived support from the recommender, and the overall satisfaction with chosen measures. Moreover, we controlled for a user's environmental concern, using the revised NEP scale [16]. Finally, users could disclose their email address to receive information on chosen measures. 


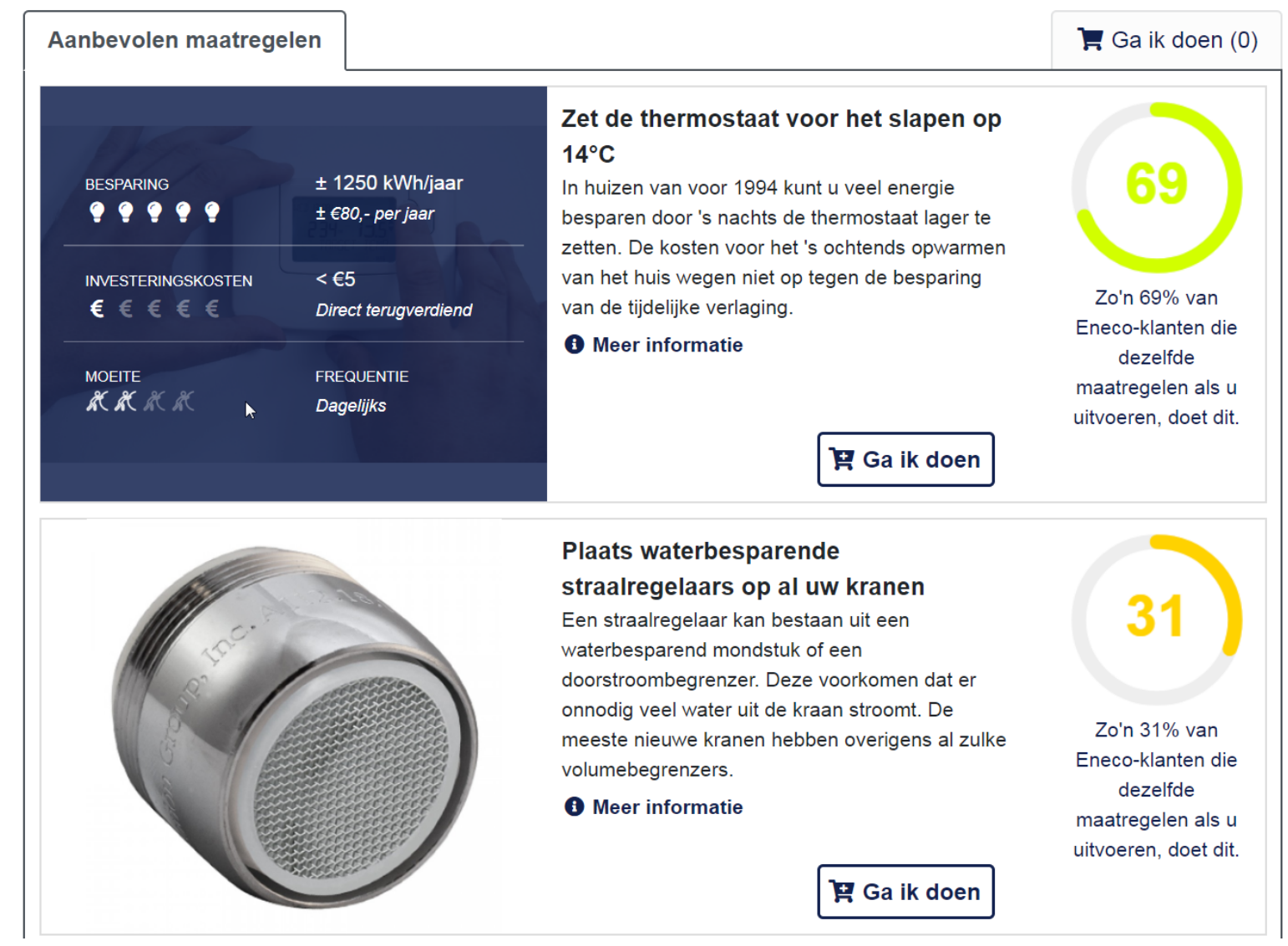

Figure 1. Our Dutch 'Saving Aid' energy recommender interface (NL: 'Besparingshulp.nl). Depicted are the name and a short description of the top-2 recommendations in our interface (e.g. at the top 'Lower the thermostat to $14{ }^{\circ} \mathrm{C}$ before going to bed'), out of a total of nine recommendations. Users could select any number of measures they would like to perform, by clicking 'I will do this' (NL: "Ga ik doen”). Measures are sorted from high to low kWh savings. On the left, users could hover a measure's image to inspect additional attributes: $\mathrm{kWh}$ savings (NL: 'Besparing'; scaled from 1 to 5 lightbulbs), the annual savings (in $€$ ), investment costs (NL: 'investeringskosten'), payback period (from '< $€ 5$ ' to 'never'), effort (NL: 'moeite'), and behavioral frequency (NL: 'frequentie'). Depending on the condition, the scores on the right either show a score or a norm percentage. Depicted here is a 'Similar norm' ("About X\% of Eneco customers who perform the same measures as you, do this").

\subsubsection{Research Design}

The presented score and explanation depicted alongside each measure in the list is subject to four between-subject conditions. In line with Goldstein et al. [22], we compared three norm explanations to an environmental baseline:

1. 'Savings' score (baseline): We presented a 'Saving Score' of 0 to 100 , with 100 for the highest kWh savings in the list.

2. 'Global' norm: The adoption rate of measures on the scale, explained as: "XX\% of other customers do this."

3. 'Similar' norm: The user's adoption probability (between $18 \%$ and $75 \%$ ), explained as: "XX\% of other customers who perform the same measures as you, do this."

4. 'Experienced' norm: The probability for an attitude 1 logit above the current user, which fell between $37.8 \%$ and $88 \%$. It was explained as: " $X X \%$ of customers who perform more measures than you, perform this measure."

\subsection{Measures}

\subsubsection{Attributes \& characteristics}

Our interface discerned between one main attribute and a set of secondary attributes that would be revealed by hovering an energy-saving measure. We used the presented score as a continuous measure ('Savings Score' or norm \%'s) to assess its impact on the probability that a measure was chosen [H2]. To control for the influence of other attributes, we included each measure's perceived effort (estimated in our pre-study), for it was an important determinant in energy decision-making [53].

Furthermore, to address our hypotheses [H3a, H3b] we dichotomized the user's energy-saving attitude to discern between users with weak and strong attitudes. Theoretically we would place a cut-off at $\theta=0$, but due to the relatively strong attitude of our sample (Median $=0.42, M=0.52 ; S D=0.79$ ) we placed the cut-off at $\theta \leq 0.25$, which comprised $39.6 \%$ of the sample (NB: a cut-off at the median would create rather uneven groups, 
as only a small part of the sample was above the median. However, a median cut-off led to roughly similar results).

\subsubsection{User evaluation aspects}

To address our hypotheses on the user's perception [H5-H8], we submitted all other items in our evaluation questionnaire to a confirmatory factor analysis (Table 2). We had to drop perceived support for our subsequent structural equation model (SEM) analysis, as it could not reliably discerned from the choice satisfaction aspect, violating divergent validity [36]. Both the feasibility $(\alpha=.72)$ and choice satisfaction aspects $(\alpha=.87)$ had an acceptable internal consistency and met the standards for convergence validity (AVE > 0.5) [36].

Finally, we used all 15 items from the NEP to assess a user's environmental concern. Eventually, only 6 items could be used reliably, which had an acceptable internal consistency $(\alpha=.78)$.

Table 2. Results of the confirmatory factory analysis on user experience. Items without loading were removed from the final model. Perceived Support was omitted due to high cross-loadings with Choice Satisfaction [36].

\begin{tabular}{|c|c|c|}
\hline Aspect & Item & Loading \\
\hline $\begin{array}{l}\text { Perceived } \\
\text { Feasibility }\end{array}$ & $\begin{array}{l}\text { The recommended measures are hard to } \\
\text { perform. }\end{array}$ & -.808 \\
\hline \multirow{3}{*}{$\begin{array}{l}\text { AVE: } .54 \\
\text { Alpha: } .72\end{array}$} & $\begin{array}{l}\text { I do not have the possibility to perform the } \\
\text { recommended measures. }\end{array}$ & -.566 \\
\hline & $\begin{array}{l}\text { The recommended measures are applicable } \\
\text { in my home environment. }\end{array}$ & \\
\hline & $\begin{array}{l}\text { It takes little effort to perform the } \\
\text { recommended measures. }\end{array}$ & .738 \\
\hline \multirow{7}{*}{$\begin{array}{l}\text { Perceived } \\
\text { Support }\end{array}$} & I want to use the Saving Aid more often. & \\
\hline & The Saving Aid was useless. & \\
\hline & I make better choices using the Saving Aid. & \\
\hline & & \\
\hline & $\begin{array}{l}\text { I would recommend the Saving Aid to } \\
\text { others. }\end{array}$ & \\
\hline & $\begin{array}{l}\text { The Saving Aid was useful to find } \\
\text { appropriate measures. }\end{array}$ & \\
\hline & $\begin{array}{l}\text { I could easily choose measures with the } \\
\text { Saving Aid. }\end{array}$ & \\
\hline Choice & I like the measures I've chosen. & .690 \\
\hline satisfaction & $\begin{array}{l}\text { I think I chose the best measures from the } \\
\text { list. }\end{array}$ & .659 \\
\hline AVE: .67 & $\begin{array}{l}\text { I would enjoy performing the chosen } \\
\text { measures. }\end{array}$ & .746 \\
\hline Alpha: .87 & $\begin{array}{l}\text { The chosen measures exactly fit my } \\
\text { preferences. }\end{array}$ & .820 \\
\hline
\end{tabular}

\section{Results}

We investigated to what extent depicting social norms alongside an attitude-tailored list of energy-saving measures affected choice behavior, compared to presenting a measure's kWh savings. First, we examined whether the number of chosen measures differed between conditions, as well as what measures were chosen [H1-H4]. Second, we investigated whether the different normative messages affected a user's perception of the system, compared to the kWh savings baseline [H5-H8].

\subsection{Choice behavior}

We used a multilevel logistic regression analysis to estimate the likelihood that a measure was chosen. Table 3 presents two models, each clustered at the user level. Both models examined whether more measures were chosen in a normative condition than in the baseline ('Main effects'). Furthermore, under 'Within list', Model 1 considers whether the actual value of the score (baseline) or norm percentage (treatment conditions) shown in the interface affected the probability that a measure was chosen, while Model 2 considers the score, perceived effort and the interaction between the two. All 'Within list' predictors considered the effect in the kWh savings baseline ('score', 'effort', and 'score X effort'), while and effects in the normative conditions were compared to the baseline, examining whether they differed from the baseline. This was modeled as an interaction between the predictor and a condition dummy variable.

Table 3. Two multilevel logistic regression analyses predicting the choice probability per measure, clustered at the user level. Reported are odds ratios (OR) and standard errors (S.E.) $(O R<1$ implies a negative effect, $O R>1$ a positive effect). ${ }^{* * *} p<0.001,{ }^{* *} p<0.01,{ }^{*} p<0.05$.

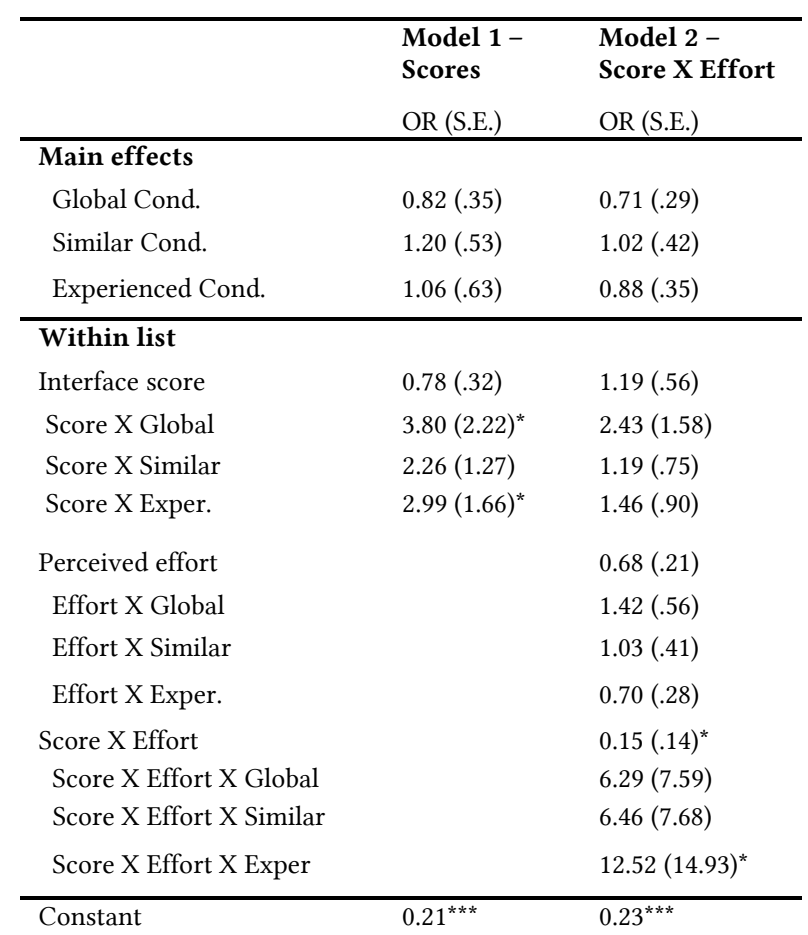

\subsubsection{Number of chosen measures per condition}

We hypothesized [H1] that users would be more likely to choose measures that were explained using social norms, rather than through their environmental benefits. Contrary to our expectations, the models in Table 3 provides no support for [H1], as the normative conditions were not significantly different from the baseline (all odds ratios (ORs) of the main effects were around 
1). This is also illustrated in Figure 2, which depicts small and nonsignificant differences in the number of chosen measures between the Savings baseline and each norm condition.

Moreover, we hypothesized [H3] that users with a weak attitude would choose more measures when facing Global norms compared to Similar norms, and vice versa for users with a strong attitude. However, a Kruskal-Wallis tests of ranks revealed no difference between Global and Similar norms for with weak attitude: $H(1,36)=0.267, p=0.61$, nor for strong attitudes: $H(1,65)$ $=0.45, p=0.50$. In addition, we hypothesized [H4] that users facing Expert norms would choose measures than those facing similar norms, but a Kruskal-Wallis tests of ranks revealed no significant difference between the two: $H(1,105)=0.028, p=0.87$. Figure 2 confirms this, for it shows no difference between norms.

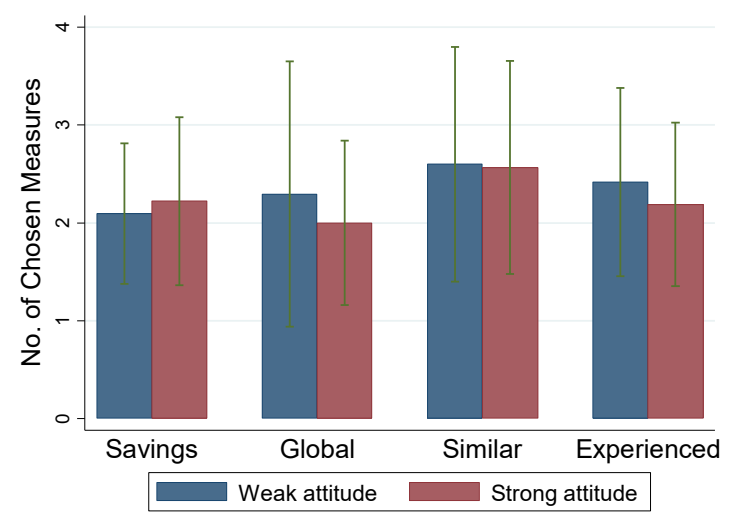

Figure 2. Total number of chosen measures, per condition and attitude strength. Error bars are 1 S.E.

\subsubsection{KWh savings vs norm percentage}

Table 3, Model 1 shows whether the presented scores affected the probability that a measure was chosen. In the baseline, we found that depicting higher Savings Scores did not affect this probability: $O R=0.78, p=0.533$, pointing out that higher $\mathrm{kWh}$ savings do not persuade users to choose a measure. The left-hand graph of Figure 3 illustrates this, as the proportion of chosen measures did not differ across kWh Savings scores.

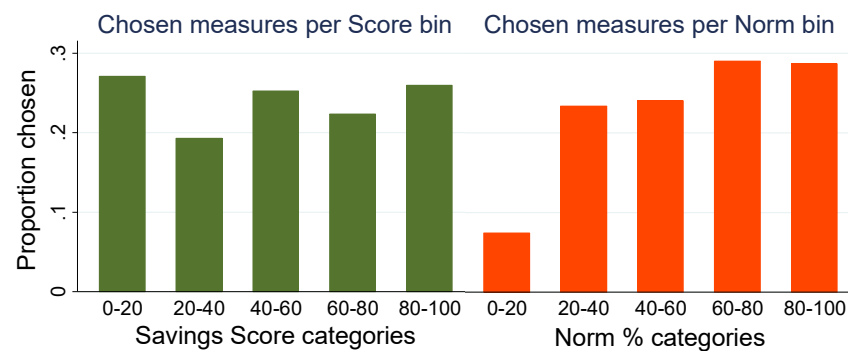

Figure 3. The left-hand side depicts the proportion of chosen measures in the baseline condition (per Savings Score category). The right-hand side does so for norm percentages (the treatment conditions), averaged across all categories (Global, Similar, and Experienced).
In contrast, Model 1 provided evidence for [H2], that showing higher norm scores increases the likelihood that a measure was chosen, compared to the effect of presenting high kWh savings. Table 3 shows that the Score in the Global $(O R=3.80, p=0.022)$ and Experienced conditions $(O R=2.99, p=0.049)$ increased the likelihood that a measure was chosen, while no such effect was found for Score in the 'Similar' condition $(O R=2.26, p=0.15)$, each compared to the baseline. This suggested that high 'Global' and 'Experienced' norm percentages were more persuasive than presenting a measure's kWh savings, while we could not make such assertions for 'Similar' norms.

Furthermore, we explored what norm percentage cohorts were more likely to persuade a user to choose a measure. The righthand side of Figure 3 shows two levels at which the proportion of chosen measures increased: around $20 \%$ (an increase from 0.07 to 0.23 ) and $60 \%$ (an increase from 0.24 to 0.29 ). This suggested that normative messages below $20 \%$ possibly discouraged users from choosing them, while those explained with percentages over $60 \%$ seemed the most likely to be chosen.

\subsubsection{Perceived effort}

As an exploratory analysis, we extended Model 1 in Table 3 by adding a measure's perceived effort as a predictor. Although users typically avoid effortful measures [53], it was possible that presenting high norm scores in our interface would overcome a measure's effort and persuade users to choose such a measure.

Table 3, Model 2 also reports the effect of perceived effort, and possible interactions with the presented score, on the probability that a measure was chosen. Compared to Model 1, the significant within-list effects for score disappeared and no effects were observed for perceived effort ( $p>0.05$ for all conditions). However, Model 2 did reveal an interaction effect between score and effort that negatively affected the likelihood that a measure was chosen in the Savings condition: $O R=0.15, p=0.041$. This suggested that measures with high $\mathrm{kWh}$ savings were more likely to be chosen if they had low levels of effort, while this likelihood decreased if a measure had high levels of perceived effort. The latter, high effort and high savings, was far more common among the set of energy-saving measures used in this study.

With regard to the effect of effort on choice in the normative conditions, Table 3, Model 2 reveals no significant differences between the effects in the baseline and the Global and Similar conditions $(O R \approx 6.3, p>0.05)$. This suggested that these norms did not persuade users to choose more effortful measures. In contrast, we did observe a significant difference the baseline's effect of effort on choice and the effect in the 'Experienced' norm condition: $O R=12.52, p=0.034$. We found that high percentages explained in terms of experienced peers increased the probability that effortful measures were chosen, rather than the low-effort ones. The odds ratio of this positive effect was two times larger than the negative effect in the baseline, showing that higher 'Experienced' norm scores could persuade users to choose effortful measures. 


\subsubsection{Conclusion}

We found that the use of descriptive norms did not persuade users to choose more energy-saving measures in total, compared to our kWh savings baseline. However, recommendations were more likely to be chosen if they were presented alongside high scores or percentages, suggesting that they stand out from a list of tailored measures. In particular, it seemed that presenting 'Experienced' norms alongside effortful measures could increase the likelihood that they were chosen, while emphasizing their kWh savings led users to choose relatively low-effort measures that typically have minor environmental benefits.

\subsection{User evaluation of interfaces \& mediation}

Finally, we examined whether perceptions of the recommender system differed between conditions, and whether this affected, in turn, choice behavior and satisfaction [H5-H8]. We organized the objective constructs, subjective constructs and relevant interactions into a path model using Structural Equation Modeling in MPlus [36, 42]. To do so, a confirmatory factor analysis was performed first (cf. Table 2), after which we tested a fully saturated model and performed stepwise removal of non-significant relations.

\subsubsection{Structural equation model}

Figure 4 depicts the final path model that had excellent fit statistics, indicating there was little unexplained variance: $\chi^{2}(63)$ $=71.548, p=0.76, C F I=1.000, T L I=1.006, R M S E A=0.000,90 \%-C I$ : $[0.000,0.028]$. The final model met the guidelines for discriminant validity [36].

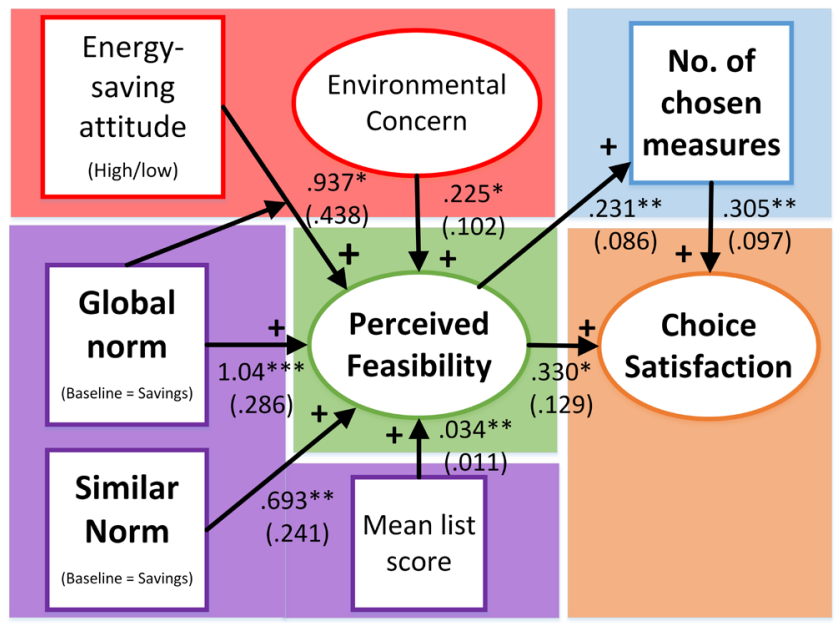

Figure 4. Structural Equation Model (SEM). Numbers on the arrows represent the $\beta$-coefficients, standard errors are between brackets. Effects between subjective constructs are standardized and resemble correlations. ${ }^{* * *} p<0.001,{ }^{* *} p<$ $0.01,{ }^{*} p<0.05$.

\subsubsection{Perceived feasibility and choice behavior}

We hypothesized that the different social norm interfaces would be perceived as more feasible than the kWh savings baseline. Figure 4 partially confirms hypothesis [H5], as both the Global norm $(\beta=1.04)$ and Similar norm conditions $(\beta=0.693)$ positively affected a recommendation list's perceived feasibility compared to the baseline. Moreover, the interaction between energy-saving attitude and Global norm on perceived feasibility ( $\beta=0.937)$ matched our expectation that the user's attitude determined how the global condition was evaluated (cf. [H3]).

In contrast, no such effect on feasibility was observed for 'Experienced' norms $(\beta=-.286, p=.310$; not in Fig. 4). Although our analysis in Table 3 suggested that 'Experienced' norms convinced users to choose effortful measures, they did not increase the perceived feasibility of the presented measures altogether.

Our final model in Figure 4 omitted perceived support due to a violation of divergence validity [36]. Hence, we could not test our hypotheses that feasibility would positively affect perceived support and, in turn, the number of chosen measures and choice satisfaction [H6-H8]. Instead, Figure 4 shows that the increased perceived feasibility due to global and similar norms directly led users to choose more measures. A (bootstrapped) test of indirect effects from the Global condition towards the number of chosen measures was significant: $\beta=0.240,95 \%$-CI: [0.020,0.460], $p=$ 0.033 , but the effect from the Similar condition to chosen measures was not significant: $\beta=0.160,95 \%-C I$ : $[-0.009,0.329], p=0.064$.

\subsubsection{Choice satisfaction}

The SEM model in Figure 4 shows two positive effects on choice satisfaction: perceived feasibility $(\beta=0.330)$ and the number of chosen measures $(\beta=0.305)$. This suggested that choosing feasible measures, as well as more measures positively affected how they were evaluated. In terms of indirect effects, the path from Global norms to choice satisfaction $(\beta=0.417,95 \%-C I$ : [0.041, 0.793], $p=0.030)$, as well as the path from the Similar norm condition $(\beta=0.278$, 95\%-CI: [0.034,0.521], $p=0.026)$ was significant, showing that the positive effects of these norms on satisfaction were mediated by feasibility.

Besides the interface effects, we found that the mean presented recommendation list score positively affected the list's feasibility perception $(\beta=0.034)$. This confirmed that higher scores, regardless of the source (i.e. kWh savings or norm), were related to higher levels of feasibility. Figure 4 also depicts that a user's environmental concern positively affected perceived feasibility ( $\beta$ $=0.225$ ), showing that users who attributed greater concern towards their role in protecting the environment, indicated that the recommended measures were more feasible to perform.

\subsubsection{Conclusion}

We examined whether user perceptions of recommendations were influenced by the presence of descriptive norms. The path model showed that 'Global' and 'Similar' norms increased the perceived feasibility of recommendations, relative to the Savings baseline, while no such effect was found for 'Experienced'. This suggested that the effectiveness of descriptive norms did not simply boil down to high percentages, but that the advice source played a role, possibly through similarity rather than expertise.

Furthermore, our path model shows that higher levels of feasibility increased both the number of chosen measures, as well 
as choice satisfaction. Our tests of indirect effects confirmed that most of the paths from 'Global' and 'Similar' norms to the 'number of chosen measures' and choice satisfaction were mediated by feasibility. This indicated that explaining energysaving recommendations in terms of normative messages affected how users perceived them (i.e. making them seem more feasible to perform) and, in turn, increased the number of measures that users had selected (i.e. a proxy for behavioral intention), as well as their choice satisfaction levels.

\section{Discussion}

We have investigated to what extent different norm nudges affect choice behavior in an energy recommender system. In doing so, we have focused on translating the findings of Goldstein et al. [22] on using descriptive norms for towel re-use to an $\mathrm{HCI}$ context. Specifically, we have investigated whether the merits of descriptive norms still apply in a more heterogeneous set of energy-saving measures.

Our results show that, in the domain of energy-saving advice, normative explanations affect user decision-making within tailored recommender interfaces. We find that that social proof [18] and implied norms can act as persuasive nudges, both through majority preferences (in this study: the presented norm percentages), as well as specific peers (in this study: the different norm sources). Such norms are found to be more effective than presenting additional information about key attributes of the recommended items (in this study: kWh savings).

\subsection{Influence of study design}

Contrasting with Goldstein et al. [22], the use of descriptive norms did not lead to an overall increase in the total number of chosen energy-saving measures, compared to a baseline that emphasized kWh savings. We discuss a number of possible causes for this different outcome through our study design.

For one, the decision contexts are different, in terms of the number of presented norms and measures. The observed behavior in Goldstein et al. [22] is rather straightforward, as it only promotes towel re-use by means of a door hanger in a hotel room. In contrast, our study comprises a set of attitude-tailored energysaving measures in a web shop study, presenting multiple descriptive norms simultaneously. While the change from a hotel room to a web-based interface might not have impacted the results, the fact that our recommender has simultaneously presented multiple measures with different norm percentages could have led users to make more comparative judgments. For example, users could have been influenced by the presented interface score rather than the norm source. This is supported by our findings that, in the Global and Similar norm conditions, measures were more likely to be chosen from recommendation lists if they had a comparatively high score, while revealing no main effect between norm conditions.

The results in our study clearly show that, contrary to what has been suggested in a meta-review [1], simply generalizing the effectiveness of descriptive norms for a simple, one-time behavior (towel re-use) to all types of measures is not a representative statement. Hence, potential adopters of a norm-based approach should seriously consider the nature of the behavior that is being promoted, whether it is energy conservation or other behaviors in the recommender domain that involve effort, such as healthy eating. While the effects of descriptive norms have been rather consistent in both movie and social recommender systems [11, 23, 46], these are domains distinct from energy and health, as their behaviors (e.g. clicks) face few behavioral thresholds (e.g. no costs).

\subsection{Differences in norms}

Besides differences in choice behavior as a result of the presented norm percentages, we also discuss to what extent the type of descriptive norms played a role. Goldstein et al. [22] propose that 'provincial' norms (i.e. 'local' norms as 'Similar' and 'Experienced' are more effective in changing user behavior than more global norms, as they share very specific and context-rich characteristics with the request recipient. While each normative condition in this study leverages some similarity with the user, the 'Global' and 'Similar' norms are arguably the most provincial, as they specifically emphasize similarities with the user. In contrast, the 'Experienced' norm also emphasizes a difference by 'performing more measures than you'.

Nonetheless, our path model shows effects consistent with a context-rich, provincial norm explanation. The Global and Similar norm conditions indicate higher levels of perceived feasibility compared to the Savings baseline, while no such effect is found for the Experienced condition. That analysis suggests that the increase in feasibility can be attributed to the norm source rather than the score, as the experienced norm had the highest percentages. This would suggest that users are influenced by the principle of 'similar others are doing it, therefore I can do this too', a more general heuristic for choice.

The relevance of this finding on feasibility lies in the indirect effects of Global and Similar norms on choice satisfaction, which are mediated by feasibility. The use of such normative explanations has not only increased the feasibility of the recommended measures, but has also led to higher levels of choice satisfaction compared to users in a kWh Savings baseline. This increase in choice satisfaction might be important to ultimately spur behavioral change, as it can, in turn, persuade users to re-use a recommender system at a later stage [36]. Moreover, previous studies have shown that higher levels of choice satisfaction lead to a higher likelihood that users actually implement chosen measures [51].

Although our 'Experienced' norms do not increase feasibility compared to the kWh savings baseline, our analysis on choice in recommendation lists (cf. section 4.1) reveals that it can boost preferences for high-effort measures. Unlike in the baseline condition, where only low-effort measures are more likely to be chosen for high kWh Saving Scores, higher Experienced norm percentages seem to boost the selection of high-effort measures. This finding nicely shows that personalized nudges might improve the effectiveness of a recommender system, as the persuasiveness of the 'Experienced' norm is specific to effortful measures. 


\subsection{Application of norms in other domains}

The current paper has employed social norms to support behavioral change for a user's 'better self' [17]. While such persuasion techniques may be somewhat paternalistic, it could be argued that in the energy domain the user ultimately benefits due to a lower energy bill and a positive environmental impact.

However, there are also domains in which norms can easily backfire or lead to arguably unethical situations. For instance, in the context of news recommender systems [5], normative explanations of news articles could reinforce partisan readership, if a user observes fellow democrats or conservatives consuming certain articles. Designers of recommender interfaces should always consider whether it could be harmful to a user if she 'follows the herd', and to what extent reinforcing such behavior through persuasive messaging exacerbates this.

However, our study has focused on nudging within a personalized list of recommendations. Since the presented items already fit a user, this might mitigate possible 'herd behavior'.

\subsection{Limitations}

There might be some concerns about the use of self-reported behavior and choice as our behavioral indicators. Although we are aware that self-report can be an inaccurate measurement method, it should not have had a large impact on the study's results, for we have only examined differences in choice behavior between randomly assigned conditions. In a similar vein, although the used sample is not representative for the broader population (it has a high proportion of males and a relatively strong energy-saving attitude), our randomly-assigned between-subject research design reduces the impact of the sample population on the results. However, it would be useful to replicate this research among a more representative population, to check whether our findings on specific normative explanations still apply.

\section{ACKNOWLEDGMENTS}

This work was made possible by XXXXXXXXXX XXX XXXXXXX XXXXXXXXXXXXXXXXX XXXXXXX XXXXXXX XXXXXXXXXXXXXXXXXXXXXXXXXXXXXXXXXXXXXXX

\section{REFERENCES}

[1] Abrahamse, W. and Steg, L. 2013. Social influence approaches to encourage resource conservation: A meta-analysis. Global Environmental Change. 23, 6 (Dec. 2013), 1773-1785. DOI:https://doi.org/10.1016/j.gloenvcha.2013.07.029.

[2] Abrahamse, W., Steg, L., Vlek, C. and Rothengatter, T. 2005. A review of intervention studies aimed at household energy conservation. Fournal of Environmental Psychology. 25, 3 (Sep. 2005), 273-291. DOI:https://doi.org/10.1016/j.jenvp.2005.08.002.

[3] Aldrovandi, S., Brown, G.D.A. and Wood, A.M. 2015. Social norms and rankbased nudging: Changing willingness to pay for healthy food. Fournal of Experimental Psychology: Applied. 21, 3 (2015), 242-254. DOI:https://doi.org/10.1037/xap0000048.

[4] Attari, S.Z., DeKay, M.L., Davidson, C.I. and de Bruin, W.B. 2011. Changing household behaviors to curb climate change: How hard can it be? Sustainability: The fournal of Record. 4, 1 (2011), 9-11.

[5] Bakshy, E., Messing, S. and Adamic, L.A. 2015. Exposure to ideologically diverse news and opinion on Facebook. Science. 348, 6239 (Jun. 2015), 1130-1132. DOI:https://doi.org/10.1126/science.aaa1160.

[6] Birnbaum, M.H. and Stegner, S.E. 1979. Source credibility in social judgment: Bias, expertise, and the judge's point of view. Fournal of Personality and Social Psychology. 37, 1 (1979), 48-74. DOI:https://doi.org/10.1037/0022-3514.37.1.48.
[7] Bonaccio, S. and Dalal, R.S. 2006. Advice taking and decision-making: An integrative literature review, and implications for the organizational sciences. Organizational Behavior and Human Decision Processes. 101, 2 (Nov. 2006), 127151. DOI:https://doi.org/10.1016/j.obhdp.2006.07.001.

[8] Bond, T.G. and Fox, C.M. 2006. Applying the Rasch Model: Fundamental Measurement in the Human Sciences, Second Edition. Psychology Press.

[9] Bonhard, P. and Sasse, M.A. 2006. 'Knowing me, knowing you' - Using profiles and social networking to improve recommender systems. BT Technology fournal. 24, 3 (Jul. 2006), 84-98. DOI:https://doi.org/10.1007/s10550-006-0080-3.

[10] Boudet, H.S., Flora, J.A. and Armel, K.C. 2016. Clustering household energysaving behaviours by behavioural attribute. Energy Policy. 92, (May 2016), 444454. DOI:https://doi.org/10.1016/j.enpol.2016.02.033.

[11] Chen, C.C., Shih, S.-Y. and Lee, M. 2016. Who should you follow? Combining learning to rank with social influence for informative friend recommendation. Decision Support Systems. 90, (Oct. 2016), 33-45. DOI:https://doi.org/10.1016/j.dss.2016.06.017.

[12] Cialdini, R.B. 2003. Crafting Normative Messages to Protect the Environment Current Directions in Psychological Science. 12, 4 (Aug. 2003), 105-109. DOI:https://doi.org/10.1111/1467-8721.01242.

[13] Cialdini, R.B. and Goldstein, and N.J. 2004. Social Influence: Compliance and Conformity. Annual Review of Psychology. 55, 1 (2004), 591-621. DOI:https://doi.org/10.1146/annurev.psych.55.090902.142015.

[14] Cialdini, R.B. and Trost, M.R. 1998. Social influence: Social norms, conformity and compliance. The handbook of social psychology, Vols. 1 and 2 (4th ed.). D.T. Gilbert, S.T. Fiske, and G. Lindzey, eds. McGraw-Hill. 151-192.

[15] Demarque, C., Charalambides, L., Hilton, D.J. and Waroquier, L. 2015. Nudging sustainable consumption: The use of descriptive norms to promote a minority behavior in a realistic online shopping environment. Journal of Environmental $\begin{array}{llll}\text { Psychology. 43, 2015), } & \text { (Sep. 166-174. }\end{array}$ DOI:https://doi.org/10.1016/j.jenvp.2015.06.008.

[16] Dunlap, R.E., Van Liere, K.D., Mertig, A.G. and Jones, R.E. 2000. New trends in measuring environmental attitudes: measuring endorsement of the new ecological paradigm: a revised NEP scale. Journal of social issues. 56, 3 (2000), 425-442.

[17] Ekstrand, M.D. and Willemsen, M.C. 2016. Behaviorism is Not Enough: Better Recommendations Through Listening to Users. Proceedings of the 10th ACM Conference on Recommender Systems (New York, NY, USA, 2016), 221-224.

[18] Festinger, L. 1954. A theory of social comparison processes. Human relations. 7 , 2 (1954), 117-140.

[19] Fornara, F., Carrus, G., Passafaro, P. and Bonnes, M. 2011. Distinguishing the sources of normative influence on proenvironmental behaviors: The role of local norms in household waste recycling. Group Processes \& Intergroup Relations. $14, \quad 5 \quad$ (Sep. DOI:https://doi.org/10.1177/1368430211408149.

[20] Gardner, G.T. and Stern, P.C. 2008. The short list: The most effective actions US households can take to curb climate change. Environment: science and policy for sustainable development. 50, 5 (2008), 12-25.

[21] Goldstein, D.G., Johnson, E.J., Herrmann, A. and Heitmann, M. 2008. Nudge Your Customers Toward Better Choices. Harvard Business Review. 86, 12 (Dec. 2008), 99-105.

[22] Goldstein, N.J., Cialdini, R.B. and Griskevicius, V. 2008. A Room with a Viewpoint: Using Social Norms to Motivate Environmental Conservation in Hotels. Fournal of Consumer Research. 35, 3 (Oct. 2008), 472-482. DOI:https://doi.org/10.1086/588568.

[23] Guy, I. 2015. Social Recommender Systems. Recommender Systems Handbook. F. Ricci, L. Rokach, and B. Shapira, eds. Springer US. 511-543.

[24] Handgraaf, M.J.J., Van Lidth de Jeude, M.A. and Appelt, K.C. 2013. Public praise vs. private pay: Effects of rewards on energy conservation in the workplace.

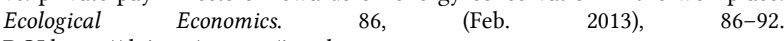
DOI:https://doi.org/10.1016/j.ecolecon.2012.11.008.

[25] Häubl, G. and Murray, K.B. 2003. Preference Construction and Persistence in Digital Marketplaces: The Role of Electronic Recommendation Agents. JOURNAL OF CONSUMER PSYCHOLOGY. 13, 1 \& 2 (2003), 75-91.

[26] Heider, F. 1958. The Psychology of Interpersonal Relations. Psychology Press.

[27] Jameson, A. 2013. Choice Architecture for Human-Computer Interaction. Foundations and Trends ${ }^{\circledR}$ in Human-Computer Interaction. 7, 1-2 (2013), 1-235. DOI:https://doi.org/10.1561/1100000028.

[28] Johnson, E.J. and Goldstein, D. 2003. Do Defaults Save Lives? Science. 302, 5649 (Nov. 2003), 1338-1339. DOI:https://doi.org/10.1126/science.1091721.

[29] Juli 2018 recordmaand voor zonnepanelen: https://www.milieucentraal.nl/nieuws/2018/juli-recordopbrengst-zonnepanelen/. Accessed: 2018-11-23.

[30] Jungermann, H. and Fischer, K. 2005. Using expertise and experience for giving and taking advice. The routines of decision making. (2005), 157-173.

[31] Kaiser, F.G., Byrka, K. and Hartig, T. 2010. Reviving Campbell's Paradigm for Attitude Research. Personality and Social Psychology Review. 14, 4 (Jan. 2010), 351-367. DOI:https://doi.org/10.1177/1088868310366452.

[32] Kaiser, F.G. and Keller, C. 2001. Disclosing situational constraints to ecological behavior: A confirmatory application of the mixed Rasch model. European 
Journal of Psychological Assessment. 17, $3 \quad$ (2001), 212-221. DOI:https://doi.org/10.1027//1015-5759.17.3.212

[33] Kaptein, M., Markopoulos, P., de Ruyter, B. and Aarts, E. 2015. Personalizing persuasive technologies: Explicit and implicit personalization using persuasion profiles. International Journal of Human-Computer Studies. 77, (May 2015), 3851. DOI:https://doi.org/10.1016/j.ijhcs.2015.01.004.

[34] Knijnenburg, B.P., Bostandjiev, S., O'Donovan, J. and Kobsa, A. 2012 Inspectability and control in social recommenders. Proceedings of the sixth ACM conference on Recommender systems (2012), 43-50.

[35] Knijnenburg, B.P., Willemsen, M. and Broeders, R. 2014. Smart sustainability through system satisfaction: Tailored preference elicitation for energy-saving recommenders. (2014)

[36] Knijnenburg, B.P. and Willemsen, M.C. 2015. Evaluating recommender systems with user experiments. Recommender Systems Handbook. Springer. 309-352.

[37] Kragt, M.E. and Bennett, J.W. 2012. Attribute Framing in Choice Experiments: How Do Attribute Level Descriptions Affect Value Estimates? Environmental and Resource Economics. 51, 1 (Jan. 2012), 43-59. DOI:https://doi.org/10.1007/s10640-011-9487-5.

[38] Kraut, R.E., Resnick, P., Kiesler, S., Burke, M., Chen, Y., Kittur, N., Konstan, J., Ren, Y. and Riedl, J. 2012. Building successful online communities: Evidence-based social design. Mit Press.

[39] Mankoff, J., Matthews, D., Fussell, S.R. and Johnson, M. 2007. Leveraging Social Networks To Motivate Individuals to Reduce Their Ecological Footprints. Proceedings of the 40th Annual Hawaii International Conference on System Sciences (Washington, DC, USA, 2007), 87-.

[40] McNee, S.M., Riedl, J. and Konstan, J.A. 2006. Being Accurate is Not Enough: How Accuracy Metrics Have Hurt Recommender Systems. CHI '06 Extended Abstracts on Human Factors in Computing Systems (New York, NY, USA, 2006), $1097-1101$.

[41] Mirsch, T., Lehrer, C. and Jung, R. 2017. Digital Nudging: Altering User Behavior in Digital Environments. Proceedings der 13. Internationalen Tagung Wirtschaftsinformatik (WI 2017) (St. Gallen, Switzerland, Feb. 2017), 634-648.

[42] Muthén, L.K. and Muthén, B. 2015. Mplus. The comprehensive modelling program for applied researchers: user's guide. 5, (2015).

[43] Nolan, J.M., Schultz, P.W., Cialdini, R.B., Goldstein, N.J. and Griskevicius, V. 2008. Normative social influence is underdetected. Personality and social psychology bulletin. 34, 7 (2008), 913-923.

[44] Schäfer, H., Hors-Fraile, S., Karumur, R.P., Calero Valdez, A., Said, A., Torkamaan, H., Ulmer, T. and Trattner, C. 2017. Towards Health (Aware) Recommender Systems. Proceedings of the 2017 International Conference on Digital Health (New York, NY, USA, 2017), 157-161.

[45] Schäfer, H. and Willemsen, M.C. 2019. Rasch-based tailored goals for nutrition assistance systems. Proceedings of the 24th International Conference on Intelligent User Interfaces - IUI '19 (Marina del Ray, California, 2019), 18-29.

[46] Sharma, A. and Cosley, D. 2013. Do social explanations work?: studying and modeling the effects of social explanations in recommender systems. Proceedings of the 22nd international conference on World Wide Web (2013), 11331144

[47] Smith, N.C., Goldstein, D.G. and Johnson, E.J. 2009. Smart Defaults: From Hidden Persuaders to Adaptive Helpers. Technical Report \#ID 1116650. Social Science Research Network.

[48] Sniezek, J.A. and Van Swol, L.M. 2001. Trust, Confidence, and Expertise in a Judge-Advisor System. Organizational Behavior and Human Decision Processes. 84, 2 (Mar. 2001), 288-307. DOI:https://doi.org/10.1006/obhd.2000.2926.

[49] Staats, H., Harland, P. and Wilke, H.A. 2004. Effecting durable change a team approach to improve environmental behavior in the household. Environment and Behavior. 36, 3 (2004), 341-367.

[50] Starke, A. 2019. RecSys Challenges in achieving sustainable eating habits. (Copenhagen, Denmark, Sep. 2019), 29-30.

[51] Starke, A., Willemsen, M. and Snijders, C. 2017. Effective User Interface Design to Increase Energy-efficient Behavior in a Rasch-based Energy Recommender System. Proceedings of the Eleventh ACM Conference on Recommender Systems (New York, NY, USA, 2017), 65-73.

[52] Starke, A., Willemsen, M.C. and Snijders, C. 2015. Saving Energy in 1-D Tailoring Energy-saving Advice Using a Rasch-based Energy Recommender System. DMRS (2015), 5-8.

[53] Starke, A.D. 2019. Supporting energy-efficient choices using Rasch-based recommender interfaces. (2019).

[54] Thaler, R.H. and Sunstein, C.R. 2008. Nudge: Improving decisions about health, wealth, and happiness. Yale University Press.

[55] Tintarev, N. and Masthoff, J. 2012. Evaluating the Effectiveness of Explanations for Recommender Systems. User Modeling and User-Adapted Interaction. 22, 4-5 (Oct. 2012), 399-439. DOI:https://doi.org/10.1007/s11257-011-9117-5.

[56] Tomkins, S., Isley, S. and Getoor, L. 2018. Sustainability at Scale: Towards Bridging the Intention-Behavior Gap with Sustainable Recommendations. Proceedings of the 12th ACM Conference on Recommender Systems (Vancouver, British Columbia, Canada, 2018), 214-218.
[57] Urban, J. and Ščasný, M. 2016. Structure of Domestic Energy Saving: How Many Dimensions? Environment and Behavior. 48, 3 (Apr. 2016), 454-481. DOI:https://doi.org/10.1177/0013916514547081.

[58] Verhallen, T.M.M. and Pieters, R.G.M. 1984. Attitude theory and behavioral costs. Journal of Economic Psychology. 5, 3 (Sep. 1984), 223-249. DOI:https://doi.org/10.1016/0167-4870(84)90024-2.

[59] Yaniv, I., Choshen-Hillel, S. and Milyavsky, M. 2011. Receiving advice on matters of taste: Similarity, majority influence, and taste discrimination. Organizational Behavior and Human Decision Processes. 115, 1 (May 2011), 111120. DOI:https://doi.org/10.1016/j.obhdp.2010.11.006.

[60] Zhu, H. and Huberman, B.A. 2014. To Switch or Not To Switch: Understanding Social Influence in Online Choices. American Behavioral Scientist. 58, 10 (Sep. 2014), 1329-1344. DOI:https://doi.org/10.1177/0002764214527089. 